\title{
Reflets
}

Revue ontaroise d'intervention sociale et communautaire

\section{La violence démasquée}

\section{Thérèse Grandmont}

Volume 2, numéro 1, printemps 1996

Contrer la violence subie par les femmes et les enfants en milieu familial

URI : https://id.erudit.org/iderudit/026111ar

DOI : https://doi.org/10.7202/026111ar

Aller au sommaire du numéro

Éditeur(s)

Reflets : Revue ontaroise d'intervention sociale et communautaire

ISSN

1203-4576 (imprimé)

1712-8498 (numérique)

Découvrir la revue

Citer cet article

Grandmont, T. (1996). La violence démasquée. Reflets, 2(1), 146-154.

https://doi.org/10.7202/026111ar

Tous droits réservés (C) Reflets : Revue ontaroise d'intervention sociale et communautaire, 1996

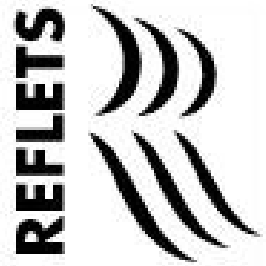

Ce document est protégé par la loi sur le droit d'auteur. L'utilisation des services d'Érudit (y compris la reproduction) est assujettie à sa politique d'utilisation que vous pouvez consulter en ligne.

https://apropos.erudit.org/fr/usagers/politique-dutilisation/ 


\section{La violence démasquée}

\section{T hérèse $\mathrm{G}$ randmont}

C oordonnatrice provindale F F C F de l'O ntario

Voilà déjà plusieurs années, que la violence faite aux femmes est I'objet de nombreuses initiatives au Canada. II y a environ dix ans, on rapportait qu'une $C$ anadienne sur dix était victime d'un acte de violence quelconque. Les études ont depuis beaucoup évolué. II en ressort que le nombre réel des femmes victimes de violence est beaucoup plus élevé qu'on n'ose l'avouer.

Dans son désir de s'engager dans la lutte contre la violence faite aux femmes, la FFCF de l'O ntario a, elle aussi, cherché à mieux comprendre ce problème social. Son projet de rechercheaction, mené de 1990 à 1992, a permis de cerner la situation des 0 ntariennes francophones âgées de 45 à 64 ans. D ans le rapport final de ce projet, intitulé C essons de penser que l'amour va tout vaincre, on révèle que plus d'une répondante sur cinq a admis avoir subi une ou plusieurs formes de violence durant l'année du sondage ( $G$ arceau et al., 1992).

C'est au printemps 1992 que débutait le projet de la V iolence démasquée. II avait pour mission de démasquer et de dénoncer la violence sous toutes ses formes, dès son apparition et dans toutes situations quelles qu'elles soient.

\section{Objectifs et modalités du projet}

Les membres du Conseil d'administration de la FFCF de l'O ntario désiraient concevoir un projet qui réussirait à atteindre les objectifs suivants: 
1. Susciter des occasions permettant aux femmes francophones de prendre conscience des différentes manifestations de violence qui existent ou qui pourraient exister dans leurs relations actuelles ou futures.

2. Aider les femmes francophones à acquérir une certaine compréhension de la violence faite aux femmes.

3. Amorcer chez les femmes francophones la prise en charge de leur situation, afin qu'elles puissent mettre en oeuvre un processus de changement si besoin est, ou qu'elles soient en mesure de garder leur environnement sans violence.

4. A mener les femmes francophones à identifier les ressources à leur disposition dans leur milieu, et à connaître les démarches à suivre pour obtenir de l'aide.

5. Encourager les femmes francophones à affirmer leur responsabilité sociale face à la violence.

Le moyen choisi pour atteindre ces objectifs a été de concevoir et diffuser une trousse $L$ a violence démasquée ${ }^{1}$. C elle-ci comprend une vidéo-cassette avec un cahier de réflexion et d'animation, permettant la mise au grand jour et la dénonciation d'attitudes sociale face à la violence,et des manifestations de la violence sous toutes ses formes. La vidéo-cassette présente trois monologues où l'on parle de diverses situations violentes, pour susciter chez les femmes une réflexion et une prise de conscience de ce qu'est la violence. C ette vidéo- cassette n'apporte pas de solutions «toutes faites», mais le visionnement provoque un moment propice à la discussion sur le problème de la violence faite aux femmes.

Le cahier de réflexion sert à appuyer les éléments soulevés dans les monologues. De plus il aide au cheminement des femmes vers une meilleure compréhension du problème, et offre quelques solutions afin de contrer la violence.

C es outils sont conçus pour être utilisés en atelier, et permettre aux personnes qui s'en servent de soutenir les femmes dans leur cheminement. 


\section{Étapes de réalisation du projet}

Pour arriver à exécuter ce projet², la fFCF de l'O ntario a choisi de le diviser en trois phases: recherche et conception des monologues, production de la vidéo et du cahier d'animation, diffusion de l'outil par la mise sur pied d'ateliers.

\section{Recherche et rédaction des monologues}

Pour commencer, nous avons effectué une recherche afin de nous documenter et, par la suite créer trois monologues. Des renseignements ont été compilés sur la violence vécue par desfemmes de différents groupes d'âge, dans les domaines sexuel, physique, verbal, psychologique, économique, professionnel, etc. N otre recherchiste/ monologuiste a ensuite procédé à la création des trois monologues se référant à ces diverses formes de violence.

Par la suite, pour vérifier si les textes des monologues représentait bien le vécu de certaines femmes, nous avons organisé des rencontres de consultation auprès d'intervenantes qui travaillent directement auprès des femmes qui vivent de la violence. $\mathrm{N}$ ous avons aussi demandé aux intervenantes de nous aider à découvrir tout ce qui pouvait manquer dans la présentation de la vidéo, et ce qui devait figurer au contenu du cahier d'animation.

\section{Mise en scène et production de la vidéo-cassette}

À partir des monologues, nous avons ensuite produit un film vidéo. Les monologues ont été révisés et adaptés selon les commentaires recueillis auprès des intervenantes communautaires. 
A près avoir recruté trois comédiennes de la région de Sudbury, nous avons aussi retenu les services d'une metteure en scène qui a travaillé avec les comédiennes pour assurer la production de la vidéo. Le tournage de la vidéo a été effectué au début du mois de décembre 1992 et le montage de production des premières copies de travail a été fait en janvier 1993.

Par la suite, nous avons entrepris un processus de rencontres et de consultations dans quelques endroits de la province afin de voir comment les femmes accueilleraient la vidéo. D éjà, nous avions reçu des indications que l'outil vidéo suscitait des sentiments divers, la prise de conscience, les réflexions, beaucoup de discussions et de mises en question. C eci nous a fait voir l'importance de bien préparer le texte de l'animation, de bien concevoir l'ensemble de l'atelier, et de bien recruter les animatrices/ facilitatrices.

J'ai beaucoup apprédé la vidéo, on a présenté trois personnes vivant trois «réalités» ficives mais drôlement vraies! (commentaire d'une partiaipante, atelier présenté à T immins).

Je n'ai pas apprécié la vidéo parce quej'ai trouvé que c'était trop proche de ma propre situation. (commentaire d'une participante, atelier présenté à $\mathrm{H}$ anmer).

Superbe, la vidéo touche vraiment le véa émotif des femmes, le mien aussi comme femme; la discussion qui s'en suit le reflète. (commentaire d'une partidpante, atelier présenté à 0 ttawa).

Je ressens un sentiment de rage, et je revis mon enfance lorsque je vois ce genre de film. (commentaire d'une participante, atelier présenté à $M$ attawa).

Je crois que cette vidéo est au rythme des personnes qui ont appris à ne jamais nommer... par ex emple. pour des personnes d'une autre génération. (commentaire d'une participante, atelier présenté à 0 ttawa). 


\section{Conception du cahier d'animation et du matériel publicitaire}

A fin de compiler les informations nécessaires à la production du cahier d'animation et de réflexion qui devait accompagner la vidéo, les services d'une responsable de projet ont été retenus. Encore ici, après consultations, les grandes lignes du contenu de notre cahier ont été tracées. N ous voulions un outil facile d'accès. II devait à la fois, servir de document d'appui pour fournir certaines informations (statistiques, définitions, causes, effets, etc.), être utile pour l'animation des ateliers, et fournir des pistes facilitant l'organisation technique des ateliers de sensibilisation.

\section{Diffusion de l'outil, et tenue des ateliers de sensibilisation et d'information}

La troisième étape du projet s'est concrétisée par la présentation aux membres de la FFCP de l'O ntario, dans toute la province, de I'atelier La violence démasquée. Toutefois, nous avons aussi pris contact avec des organismes et des groupements dans d'autres localités, susceptibles de recevoir cet atelier ${ }^{3}$.

Les sessions présentées avec les outils pédagogiques durent environ troisheureset couvrent de façon générale la problématique de la violence faite aux femmes à partir des éléments suivants: définitions, données statistiques, cycle, escalade, causes, déclencheurs, aide à la victime et ressources communautaires.

J'ai beaucoup apprécé la vidéo, dans un sens parce qu'elle dédenche la discussion et permet de réaliser certains aspects de la violence qu' on ne voit pas toujours (commentaire d'une participante, atelier présenté à F auquier). 
$M$ a première réadion: ça concerne les femmes de tous les âges, c'est triste de s'imaginer qu' on connaît probablement un homme violent. (commentaire d'une participante, atelier présenté à Sudbury).

\section{Succès du projet}

U n des grand succès de ce projet est sans contredit d'avoir réussi à rejoindre, par la présentation desateliers, plus de 825 personnes, pour la plupart des femmes et quelques hommes. Parmi ces personnes, 679 habitent le $\mathrm{N}$ ord et sont dispersées dans une trentaine de villes et villages.

Lors des ateliers, les témoignages des femmes présentes ont rendu cette partie du projet très enrichissante pour lesanimatrices ${ }^{5}$ et la FFCF de l'O ntario. M ême si nous avions parfois le sentiment de ne pouvoir intervenir directement dans certaines situations, nous avions quand même la certitude d'avoir fait une différence. $\mathrm{N}$ ous pouvions vraiment ressentir que grâce à cet atelier quelque chose avait changé chez les participantes. $N$ ous sentions qu'il s'était formé une solidarité entre femmes, ainsi que le goût de passer à l'action pour améliorer le sort de la femme violentée de 45 à 64 ans, et de toutes les femmes.

Je ne pouvais pas croire auparavant que cela pouvait exister, maintenant je sais que je pourrais peut-être aider quelqu' un. (commentaire d'une participante, atelier présenté à 0 ttawa).

$M$ a première réaction est «H ourra!», enfin on $s^{\prime}$ aperçoit de ce qui se passe. (commentaire d'une partidpante, atelier présenté à 0 ttawa).

J'ai trouvé très impressionnant de voir quelqu'un parler tout haut de ce qu'elle ressentait en dedans. $L$ a 
culpabilité et la honte font de nouveau surface à plusieurs reprises $A$ yant été victime d'inceste moi-même je trouve que c'était très réaliste. (commentaire d'une partiajpante, atelier présenté à $M$ attice).

De plus, cette étape du projet a vraiment confirmé que la trousse allait rejoindre les femmes dans leur vécu. $L$ a violence démasquée est un instrument de plus pour dévoiler la violence faite aux femmes. C'est un outil facile à utiliser, qui permet ensuite aux femmes de réagir et d'exprimer comment elles vivent cette réalité. II prépare aussi les femmes à chercher d'autres sources d'informations qui peuvent leur permettre d'approfondir les diverses composantes de cette problématique.

Au début du projet, l'intention originale était de produire une trousse de sensibilisation et de diffuser cet outil. C ependant, au fur et à mesure que le projet a avancé, il est devenu évident que notre démarche ne pouvait pas seulement se situer à ce niveau. L'évaluation de l'importance des besoins au niveau de la sensibilisation, et du manque flagrant de ressources, nousa permis de rendre notre démarche plus complète. $\mathrm{N}$ ous avons donc mis sur pied les ateliers, et pour transmettre l'information recueillie, nous sommes allées directement dans les communautés.

L ors de cette session, j' ai appris l'importance de s'affirmer contre la violence; j'ai bien aidé ma fille qui était dans une telle situation. (commentaire d'une participante, atelier présenté à $\mathrm{K}$ irkland Lake).

\section{Suivi au projet: former des formatrices}

Avec la participation de l'A ction ontarienne contre la violence faite aux femmes (AO CVF), en juin 1995, une session de formation a été organisée à Sudbury. Elle a permis de former un groupe d'une vingtaine de femmesqui sont en mesure d'utiliser la trousse La violence démasquée, et d'être plus actives dans le dossier de la violence faite aux femmes. $\mathrm{N}$ otre objectif était de permettre à ces femmes de devenir des intervenantes/ formatrices dans leur région, en approfondissant leurs connaissances, grâce à un atelier 
qui comprenait des sujets reliés à la violence. $N$ ous avons donc formé ces femmes afin qu'elles soient mieux outillées et plus à I'aise dans leurs interventions dans la communauté.

Les participantes sont venues de tous les coins de la province. $D$ ans la réalisation de cette formation, nous comptons sur l'effet multiplicateur. En effet, ces femmes deviennent celles qui dans leurs localités permettront de sensibiliser d'autres femmes ou groupes au problème de la violence qui s'exerce contre elles.

\section{Conclusion}

Le but de la FFCF de l'O ntario de démasquer la violence, nous l'avons vraiment atteint quand nous avons développé cet outil d'intervention. $N$ ous étions loin de nous imaginer à quel point cela deviendrait un défi. Ce projet nous a donné l'occasion de nous renseigner sur la problématique de la violence contre les femmes, et de rencontrer d'autres femmes qui oeuvrent à l'élimination de ce phénomène. $\mathrm{N}$ ous sommes très fières d'avoir réussi à créer un outil qui reflète la réalité des FrancoO ntariennes, et qui pourra servir longtemps à donner aux femmes une occasion de se raconter.

\section{Notes}

1. La trousse de sensibilisation $L$ a violence démasquée est en vente à un coût minime auprès de la Fédération des femmes canadiennesfrançaises de l'O ntario. Vous pouvez la commander en composant le (705) 671-1409.

2. U n projet de cette envergure n'a pu se réaliser sans la contribution et la collaboration de nombreuses personnes, organismes ou ministères. Plus particulièrement, ce projet a été concrétisé grâce au soutien financier de I'O ffice des affaires francophones de I'O ntario, par l'octroi d'une subvention substantielle s'échelonnant sur une période de trois ans, ainsi que par le Secrétariat 
d'État du Canada, programmes des langues officielles et de la femme, dont l'apport financier a permisà la FFC F de l'O ntario de poursuivre ses opérations et réaliser ce projet.

3. U ne subvention supplémentaire obtenue auprès du ministère du D éveloppement du $\mathrm{N}$ ord et des mines a permis d'assurer de façon plus intense la diffusion de la trousse dans différentes communautés isolées du nord de l'O ntario.

4. N otre atelier a été présenté aux endroits suivants: $O$ shawa,Vanier, $\mathrm{O}$ ttawa, $\mathrm{O}$ rléans, $\mathrm{H}$ aileybury, T immins, $\mathrm{H}$ earst, Pointe- auxR oches, Toronto, Cornwall, Sudbury, $\mathrm{H}$ anmer, M attice, Dubreuilville, W hite $\mathrm{R}$ iver, Chapleau, $\mathrm{M}$ anitouwadge, Wawa, SaultSte- $M$ arie, $M$ arathon, A zilda, K irkland Lake, Porcupine, R amore, Virginiatown, $\mathrm{N}$ ew Liskeard, Chelmsford, Cochrane, H oltyre, Iroquois Falls, Espanola, Elliott Lake, Sturgeon Falls, $M$ attawa, R iver Valley, Fauquier et Thunder Bay (les femmes d'I gnace, Geralton, Longlac et $\mathrm{N}$ akina étaient présentes lors de ce dernier atelier).

5. N ous avons bénéficié des services d'une équipe d'animation composée de : $G$ aëtane Pharand (Sudbury),T hérèse $G$ randmont (Sudbury), D anielle Alary (Sudbury), R enée M allet (Sudbury), Eva M cG rath G odbout (T immins) et Gilberte B risson (T immins).

\section{Bibliographie}

GAR CEAU,M .-L., D. DEN N IE, B.TR EM BLAY-M ATTE et M. CHAR RO N, (1992). C essons de penser que l'amour va tout vaincre. $L$ a situation des femmes francophones de 45 à 64 ans qui vivent en 0 ntario. R apport final, Sudbury, Fédération des femmes canadiennes françaises de l'O ntario. 